\title{
Antiproton Flux, Antiproton-to-Proton Flux Ratio, and Properties of Elementary Particle Fluxes in Primary Cosmic Rays Measured with the Alpha Magnetic Spectrometer on the International Space Station
}

M. Aguilar, ${ }^{26}$ L. Ali Cavasonza, ${ }^{1}$ B. Alpat ${ }^{32}$ G. Ambrosi,${ }^{32}$ L. Arruda,${ }^{24}$ N. Attig, ${ }^{21}$ S. Aupetit, ${ }^{17}$ P. Azzarello, ${ }^{16}$ A. Bachlechner, ${ }^{1}$ F. Barao, ${ }^{24}$ A. Barrau,${ }^{17}$ L. Barrin, ${ }^{15}$ A. Bartoloni,${ }^{38}$ L. Basara, ${ }^{36}$ S. Başeğmez-du Pree,${ }^{6}$ M. Battarbee ${ }^{46}$ R. Battiston, ${ }^{36,37, a}$ J. Bazo, ${ }^{32, b}$ U. Becker, ${ }^{9}$ M. Behlmann, ${ }^{9}$ B. Beischer, ${ }^{1}$ J. Berdugo, ${ }^{26}$ B. Bertucci, ${ }^{32,33}$ V. Bindi, ${ }^{19}$ G. Boella, ${ }^{28,29}$ W. de Boer, ${ }^{22}$ K. Bollweg, ${ }^{20}$ V. Bonnivard, ${ }^{17}$ B. Borgia, ${ }^{38,39}$ M. J. Boschini, ${ }^{28}$ M. Bourquin, ${ }^{16}$ E. F. Bueno, ${ }^{40}$ J. Burger, ${ }^{9}$ F. Cadoux,${ }^{16}$ X. D. Cai, ${ }^{9}$ M. Capell, ${ }^{9}$ S. Caroff, ${ }^{3}$ J. Casaus, ${ }^{26}$ G. Castellini, ${ }^{14}$ I. Cernuda,${ }^{26}$ F. Cervelli, ${ }^{34}$ M. J. Chae, ${ }^{41}$ Y. H. Chang, ${ }^{10}$ A. I. Chen, ${ }^{9}$ G. M. Chen, ${ }^{6}$ H. S. Chen, ${ }^{6}$ L. Cheng, ${ }^{42}$ H. Y. Chou, ${ }^{10}$ E. Choumilov, ${ }^{9}$ V. Choutko, ${ }^{9}$ C. H. Chung, ${ }^{1}$ C. Clark ${ }^{20}$ R. Clavero, ${ }^{23}$ G. Coignet, ${ }^{3}$ C. Consolandi, ${ }^{19}$ A. Contin,${ }^{7,8}$ C. Corti, ${ }^{19}$ B. Coste,${ }^{36}$ W. Creus, ${ }^{10}$ M. Crispoltoni, ${ }^{32,33,15}$ Z. Cui, ${ }^{42}$ Y. M. Dai, ${ }^{5}$ C. Delgado, ${ }^{26}$ S. Della Torre, ${ }^{28}$ M. B. Demirköz, ${ }^{2}$ L. Derome, ${ }^{17}$ S. Di Falco, ${ }^{34}$ F. Dimiccoli, ${ }^{36,37}$ C. Díaz, ${ }^{26}$ P. von Doetinchem ${ }^{19}$ F. Dong, ${ }^{30}$ F. Donnini, ${ }^{32,33}$ M. Duranti, ${ }^{32,33}$ D. D'Urso, ${ }^{32, c}$ A. Egorov, ${ }^{9}$ A. Eline, ${ }^{9}$ T. Eronen, ${ }^{46}$ J. Feng, ${ }^{45, d}$ E. Fiandrini,${ }^{32,33}$ E. Finch, ${ }^{31}$ P. Fisher, ${ }^{9}$ V. Formato, ${ }^{32,15}$ Y. Galaktionov, ${ }^{9}$ G. Gallucci, ${ }^{34}$ B. García, ${ }^{26}$ R. J. García-López, ${ }^{23}$ C. Gargiulo, ${ }^{15}$ H. Gast, ${ }^{1}$ I. Gebauer, ${ }^{22}$ M. Gervasi, ${ }^{28,29}$ A. Ghelfi, ${ }^{17}$ F. Giovacchini, ${ }^{26}$ P. Goglov, ${ }^{9}$ D. M. Gómez-Coral, ${ }^{27}$ J. Gong,${ }^{30}$ C. Goy,${ }^{3}$ V. Grabski, ${ }^{27}$ D. Grandi, ${ }^{28}$ M. Graziani,${ }^{32,33}$ I. Guerri, ${ }^{34,35}$ K. H. Guo, ${ }^{18}$ M. Habiby, ${ }^{16}$ S. Haino, ${ }^{45}$ K. C. Han,${ }^{25}$ Z. H. He, ${ }^{18}$ M. Heil,${ }^{9}$ J. Hoffman, ${ }^{19}$ T. H. Hsieh, ${ }^{9}$ H. Huang, ${ }^{45, \mathrm{e}}$ Z. C. Huang, ${ }^{18}$ C. Huh,${ }^{13}$ M. Incagli, ${ }^{34}$ M. Ionica, ${ }^{32}$ W. Y. Jang, ${ }^{13}$ H. Jinchi, ${ }^{25}$ S. C. Kang, ${ }^{13}$ K. Kanishev, ${ }^{36,37}$ G. N. Kim, ${ }^{13}$ K. S. Kim,${ }^{13}$ Th. Kirn, ${ }^{1}$ C. Konak, ${ }^{2}$ O. Kounina, ${ }^{9}$ A. Kounine, ${ }^{9}$ V. Koutsenko, ${ }^{9}$ M. S. Krafczyk, ${ }^{9}$ G. La Vacca, ${ }^{28}$ E. Laudi, ${ }^{15}$ G. Laurenti, ${ }^{7}$ I. Lazzizzera, ${ }^{36,37}$ A. Lebedev, ${ }^{9}$ H. T. Lee, ${ }^{44}$ S. C. Lee, ${ }^{45}$ C. Leluc, ${ }^{16}$ H. S. Li, ${ }^{43}$ J. Q. Li,${ }^{9, f}$ J. Q. Li, ${ }^{30}$ Q. Li ${ }^{30}$ T. X. Li,${ }^{18}$ W. Li, ${ }^{4}$ Z. H. Li,${ }^{6}$ Z. Y. Li ${ }^{45, d}$ S. Lim,${ }^{13}$ C. H. Lin, ${ }^{45}$ P. Lipari, ${ }^{38}$ T. Lippert, ${ }^{21}$ D. Liu, ${ }^{45}$ Hu Liu ${ }^{26, g}$ S. Q. Lu ${ }^{45, d}$ Y. S. Lu, ${ }^{6}$ K. Luebelsmeyer, ${ }^{1}$ F. Luo, ${ }^{42}$ J. Z. Luo ${ }^{30}$ S. S. Lv ${ }^{18}$ R. Majka,${ }^{31}$ C. Mañá, ${ }^{26}$ J. Marín, ${ }^{26}$ T. Martin, ${ }^{20}$ G. Martínez, ${ }^{26}$ N. Masi, ${ }^{7}$ D. Maurin, ${ }^{17}$ A. Menchaca-Rocha, ${ }^{27}$ Q. Meng, ${ }^{30}$ D. C. Mo, ${ }^{18}$ L. Morescalchi,${ }^{34, h}$ P. Mott, ${ }^{20}$ T. Nelson, ${ }^{19}$ J. Q. Ni, ${ }^{18}$ N. Nikonov, ${ }^{1}$ F. Nozzoli,${ }^{32, c}$ P. Nunes, ${ }^{24}$ A. Oliva, ${ }^{26}$ M. Orcinha,${ }^{24}$ F. Palmonari, ${ }^{7,8}$ C. Palomares ${ }^{26}$ M. Paniccia, ${ }^{16}$ M. Pauluzzi ${ }^{32,33}$ S. Pensotti, ${ }^{28,29}$ R. Pereira, ${ }^{19}$ N. Picot-Clemente, ${ }^{12}$ F. Pilo,${ }^{34}$ C. Pizzolotto, ${ }^{32, c}$ V. Plyaskin, ${ }^{9}$ M. Pohl, ${ }^{16}$ V. Poireau, ${ }^{3}$ A. Putze, ${ }^{3, i}$ L. Quadrani, ${ }^{7,8}$ X. M. Qi ${ }^{18}$ X. Qin, ${ }^{32, j}$ Z. Y. Qu, ${ }^{45, k}$ T. Räihä, ${ }^{1}$ P. G. Rancoita, ${ }^{28}$ D. Rapin, ${ }^{16}$ J. S. Ricol, ${ }^{17}$ I. Rodríguez, ${ }^{26}$ S. Rosier-Lees, ${ }^{3}$ A. Rozhkov, ${ }^{9}$ D. Rozza, ${ }^{28}$ R. Sagdeev, ${ }^{11}$ J. Sandweiss, ${ }^{31}$ P. Saouter, ${ }^{16}$ S. Schael, ${ }^{1}$ S. M. Schmidt, ${ }^{21}$ A. Schulz von Dratzig, ${ }^{1}$ G. Schwering, ${ }^{1}$ E. S. Seo, ${ }^{12}$ B. S. Shan, ${ }^{4}$ J. Y. Shi, ${ }^{30}$ T. Siedenburg, ${ }^{1}$ D. Son, ${ }^{13}$ J. W. Song, ${ }^{42}$ W. H. Sun,,${ }^{9,1}$ M. Tacconi,${ }^{28}$ X. W. Tang, ${ }^{6}$ Z. C. Tang, ${ }^{6}$ L. Tao, ${ }^{3}$ D. Tescaro, ${ }^{23}$ Samuel C. C. Ting,,${ }^{9,15}$ S. M. Ting, ${ }^{9}$ N. Tomassetti, ${ }^{17}$ J. Torsti ${ }^{46}$ C. Türkoğlu, ${ }^{2}$ T. Urban, ${ }^{20}$ V. Vagelli, ${ }^{32}$ E. Valente, ${ }^{38,39}$ C. Vannini,${ }^{34}$ E. Valtonen, ${ }^{46}$ M. Vázquez Acosta, ${ }^{23}$ M. Vecchi, ${ }^{40}$ M. Velasco, ${ }^{26}$ J. P. Vialle, ${ }^{3}$ V. Vitale, ${ }^{32, c}$ S. Vitillo, ${ }^{16}$ L. Q. Wang, ${ }^{42}$ N. H. Wang, ${ }^{42}$ Q. L. Wang, ${ }^{5}$ X. Wang, ${ }^{9}$ X. Q. Wang, ${ }^{6}$ Z. X. Wang, ${ }^{18}$ C. C. Wei, ${ }^{45, m}$ Z. L. Weng, ${ }^{9}$ K. Whitman, ${ }^{19}$ J. Wienkenhöver, ${ }^{1}$ M. Willenbrock, ${ }^{9} \mathrm{H}$. Wu, ${ }^{30}$ X. Wu, ${ }^{16}$ X. Xia, ${ }^{26, j}$ R. Q. Xiong, ${ }^{30}$ W. Xu, ${ }^{9}$ Q. Yan, ${ }^{9}$ J. Yang, ${ }^{41}$ M. Yang, ${ }^{6}$ Y. Yang, ${ }^{43}$ H. Yi, ${ }^{30}$ Y. J. Yu, ${ }^{5}$ Z. Q. Yu, ${ }^{6}$ S. Zeissler, ${ }^{22}$ C. Zhang, ${ }^{6}$ J. Zhang, ${ }^{9, e}$ J. H. Zhang, ${ }^{30}$ S. D. Zhang, ${ }^{9, f}$ S. W. Zhang, ${ }^{6}$ Z. Zhang, Z. M. Zheng, ${ }^{4}$ Z. Q. Zhu, ${ }^{9, n}$ H. L. Zhuang, ${ }^{6}$ V. Zhukov, ${ }^{1}$ A. Zichichi, ${ }^{7,8}$ N. Zimmermann, ${ }^{1}$ and P. Zuccon ${ }^{9}$

(AMS Collaboration)

\author{
${ }^{1}$ I. Physics Institute and JARA-FAME, RWTH Aachen University, D-52056 Aachen, Germany \\ ${ }^{2}$ Department of Physics, Middle East Technical University (METU), 06800 Ankara, Turkey \\ ${ }^{3}$ Laboratoire d'Annecy-le-Vieux de Physique des Particules (LAPP), CNRS/IN2P3 and Université Savoie Mont Blanc, \\ F-74941 Annecy-le-Vieux, France \\ ${ }^{4}$ Beihang University (BUAA), Beijing, 100191, China \\ ${ }^{5}$ Institute of Electrical Engineering (IEE), Chinese Academy of Sciences, Beijing, 100190, China \\ ${ }^{6}$ Institute of High Energy Physics (IHEP), Chinese Academy of Sciences, Beijing, 100049, China \\ ${ }^{7}$ INFN Sezione di Bologna, I-40126 Bologna, Italy \\ ${ }^{8}$ Università di Bologna, I-40126 Bologna, Italy \\ ${ }^{9}$ Massachusetts Institute of Technology (MIT), Cambridge, Massachusetts 02139, USA \\ ${ }^{10}$ National Central University (NCU), Chung-Li, Tao Yuan, 32054, Taiwan \\ ${ }^{11}$ East-West Center for Space Science, University of Maryland, College Park, Maryland 20742, USA \\ ${ }^{12}$ IPST, University of Maryland, College Park, Maryland 20742, USA \\ ${ }^{13}$ CHEP, Kyungpook National University, 41566 Daegu, Korea
}




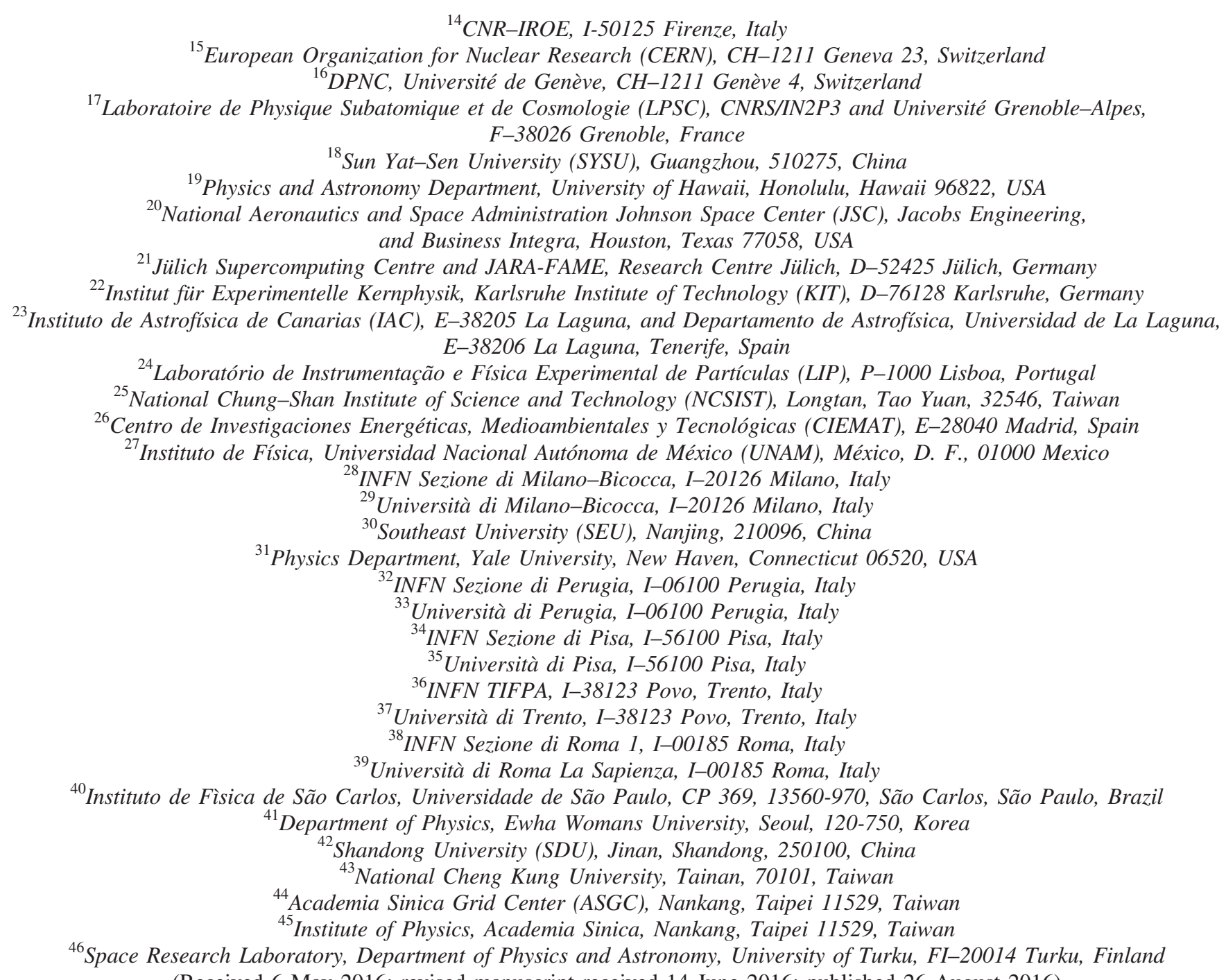

(Received 6 May 2016; revised manuscript received 14 June 2016; published 26 August 2016)

A precision measurement by AMS of the antiproton flux and the antiproton-to-proton flux ratio in primary cosmic rays in the absolute rigidity range from 1 to $450 \mathrm{GV}$ is presented based on $3.49 \times 10^{5}$ antiproton events and $2.42 \times 10^{9}$ proton events. The fluxes and flux ratios of charged elementary particles in cosmic rays are also presented. In the absolute rigidity range $\sim 60$ to $\sim 500 \mathrm{GV}$, the antiproton $\bar{p}$, proton $p$, and positron $e^{+}$fluxes are found to have nearly identical rigidity dependence and the electron $e^{-}$flux exhibits a different rigidity dependence. Below $60 \mathrm{GV}$, the $(\bar{p} / p),\left(\bar{p} / e^{+}\right)$, and $\left(p / e^{+}\right)$flux ratios each reaches a maximum. From $\sim 60$ to $\sim 500 \mathrm{GV}$, the $(\bar{p} / p),\left(\bar{p} / e^{+}\right)$, and $\left(p / e^{+}\right)$flux ratios show no rigidity dependence. These are new observations of the properties of elementary particles in the cosmos.

DOI: 10.1103/PhysRevLett.117.091103

We report on the measurement of the antiproton flux and of the antiproton-to-proton flux ratio in primary cosmic rays in the absolute rigidity range from 1 to $450 \mathrm{GV}$ based on $3.49 \times 10^{5}$ antiproton events and $2.42 \times 10^{9}$ proton

Published by the American Physical Society under the terms of the Creative Commons Attribution 3.0 License. Further distribution of this work must maintain attribution to the author(s) and the published article's title, journal citation, and DOI. events collected by the Alpha Magnetic Spectrometer, AMS, on the International Space Station, ISS, from May 19, 2011 to May 26, 2015. Of the four charged elementary particles traveling through the cosmos-protons, electrons, positrons, and antiprotons - the experimental data on antiprotons are limited because for each antiproton there are approximately $10^{4}$ protons. Since the observation of antiprotons in cosmic rays [1], many studies of cosmic ray antiprotons have been performed [2-6]. However, to measure the antiproton flux to $1 \%$ accuracy requires a 
separation power of $\sim 10^{6}$. The sensitivity of antiprotons to cosmic phenomena [7-10] is complementary to the sensitivity of the measurements of cosmic ray positrons. For example, AMS has accurately measured the excess in the positron fraction to $500 \mathrm{GeV}$ [11]. This data generated many interesting theoretical models including collisions of dark matter particles [12], astrophysical sources [13], and collisions of cosmic rays [14]. Some of these models also include specific predictions on the antiproton flux and the antiproton-to-proton flux ratio in cosmic rays.

Simultaneously with these $\bar{p}$ measurements, the electron $e^{-}$and positron $e^{+}$fluxes [15] and the proton $p$ flux [16] have been analyzed by AMS. Hence, in this Letter we also report the accurate study of the rigidity dependence of elementary particle fluxes and their ratios in primary cosmic rays. These measurements, performed with the same detector, provide precise experimental information over an extended energy range in the study of elementary particles traveling through the cosmos.

Detector.- The description of the AMS detector is presented in Refs. [17,18]. All detector elements are used for particle identification in the present analysis: the silicon tracker [19], the permanent magnet [5,20], the time of flight counters TOF [21], the anticoincidence counters ACC [22], the transition radiation detector TRD [23], the ring imaging Čerenkov detector RICH [24], and the electromagnetic calorimeter ECAL [25].

To measure the rigidity $R$ (momentum per unit of charge) of cosmic rays and to differentiate between positive and negative particles, the tracker has nine layers. The first (L1) is at the top of the detector, the second (L2) just above the magnet, six (L3 to L8) within the bore of the magnet, and the last (L9) just above the ECAL. L2 to L8 constitute the inner tracker. For $|Z|=1$ particles the maximum detectable rigidity, MDR, is $2 \mathrm{TV}$ and the charge resolution is $\Delta Z=0.05$. The TOF measures $|Z|$ and velocity with a resolution of $\Delta \beta / \beta^{2}=4 \%$. The ACC has 0.99999 efficiency to reject cosmic rays entering the inner tracker from the side.

The TRD separates $\bar{p}$ and $p$ from $e^{-}$and $e^{+}$using the $\Lambda_{\text {TRD }}$ estimator constructed from the ratio of the log-likelihood probability of the $e^{ \pm}$hypothesis to that of the $\bar{p}$ or $p$ hypothesis in each layer [11]. Antiprotons and protons, which have $\Lambda_{\mathrm{TRD}} \sim 1$, are efficiently separated from electrons and positrons, which have $\Lambda_{\mathrm{TRD}} \sim 0.5$. The RICH has a velocity resolution $\Delta \beta / \beta=0.1 \%$ for $|Z|=1$ to ensure separation of $\bar{p}$ and $p$ from light particles $\left(e^{ \pm}\right.$and $\pi^{ \pm}$) below $10 \mathrm{GV}$. The ECAL is used to separate $\bar{p}$ and $p$ from $e^{-}$ and $e^{+}$when the event can be measured by the ECAL.

To distinguish antiprotons from charge confusion protons, that is, protons which are reconstructed in the tracker with negative rigidity due to the finite tracker resolution or due to interactions with the detector materials, a charge confusion estimator $\Lambda_{\mathrm{CC}}$ is defined using the boosted decision tree technique [26]. The estimator combines information from the tracker such as the track $\chi^{2} /$ d.o.f., rigidities reconstructed with different combinations of tracker layers, the number of hits in the vicinity of the track, and the charge measurements in the TOF and the tracker. With this method, antiprotons, which have $\Lambda_{\mathrm{CC}} \sim+1$, are efficiently separated from charge confusion protons, which have $\Lambda_{\mathrm{CC}} \sim-1$.

Event selection and data samples. - Over 65 billion cosmic ray events have been recorded in the first 48 months of AMS operations. Only events collected during normal detector operating conditions are used in this analysis. This includes the time periods when the AMS $z$ axis is pointing within $40^{\circ}$ of the local zenith and when the ISS is not in the South Atlantic Anomaly. Data analysis is performed in 57 absolute rigidity bins. The same binning as in our proton flux measurement [16] is chosen below 80.5 GV. Above $80.5 \mathrm{GV}$ two to four bins from Ref. [16] are combined to ensure sufficient antiproton statistics.

Events are selected requiring a track in the TRD and in the inner tracker and a measured velocity $\beta>0.3$ in the TOF corresponding to a downward-going particle. To maximize the number of selected events while maintaining an accurate rigidity measurement, the acceptance is increased by releasing the requirements on the external tracker layers, L1 and L9. Below $38.9 \mathrm{GV}$ neither L1 nor L9 is required. From 38.9 to $147 \mathrm{GV}$ either L1 or L9 is required. From 147 to $175 \mathrm{GV}$ only L9 is required. Above $175 \mathrm{GV}$ both L1 and L9 are required. In order to maximize the accuracy of the track reconstruction, the $\chi^{2} /$ d.o.f. of the reconstructed track fit is required to be less than 10 both in the bending and nonbending projections. The $d E / d x$ measurements in the TRD, the TOF, and the inner tracker must be consistent with $|Z|=1$. To select only primary cosmic rays, the measured rigidity is required to exceed the maximum geomagnetic cutoff by a factor of 1.2 for either positive or negative particles within the AMS field of view. The cutoff is calculated by backtracing [16,27] using the most recent IGRF geomagnetic model [28].

Events satisfying the selection criteria are classified into two categories-positive and negative rigidity events. A total of $2.42 \times 10^{9}$ events with positive rigidity are selected as protons. They are $99.9 \%$ pure protons with almost no background. Deuterons are not distinguished from protons, their contribution decreases with rigidity: at $1 \mathrm{GV}$ it is less than $2 \%$ and at $20 \mathrm{GV}$ it is $0.6 \%$ [5,29]. The effective acceptance of this selection for protons is larger than in our proton flux publication [16]. This is because there is no strict requirement that selected particles pass through the tracker layers L1 and L9 (see above) leading to a much larger field of view at low rigidities and, therefore, to a significant increase in the number of protons.

The negative rigidity event category comprises both antiprotons and several background sources: electrons, light negative mesons ( $\pi^{-}$and a negligible amount of $K^{-}$) produced in the interactions of primary cosmic rays 
with the detector materials, and charge confusion protons. The contributions of the different background sources vary with rigidity. For example, light negative mesons are present only at rigidities below $10 \mathrm{GV}$, whereas charge confusion becomes noticeable only at high rigidities. Electron background is present at all rigidities. The combination of information from the TRD, TOF, tracker, $\mathrm{RICH}$, and ECAL enables the efficient separation of the antiproton signal events from these background sources using a template fitting technique. The number of observed antiproton signal events and its statistical error in the negative rigidity sample are determined in each bin by fitting signal and background templates to data by varying their normalization. As discussed below, the template variables used in the fit are constructed using information from the TOF, tracker, and TRD. The distribution of the variables for the template definition is the same for antiprotons and protons if they are both reconstructed with a correct charge-sign. This similarity has been verified with the Monte Carlo simulation [30] and the antiproton and proton data of $2.97 \leq|R|<18.0 \mathrm{GV}$. Therefore, the signal template is always defined using the high-statistics proton data sample. Three overlapping rigidity regions with different types of template function are defined to maximize the accuracy of the analysis: low absolute rigidity region (1.00$4.02 \mathrm{GV})$, intermediate region (2.97-18.0 GV), and high absolute rigidity region (16.6-450 GV). In the overlapping rigidity bins, the results with the smallest error are selected.

At low rigidities, a cut on the TRD estimator $\Lambda_{\mathrm{TRD}}$ and the velocity measurement in the TOF are important to differentiate antiprotons from light particles $\left(e^{-}\right.$and $\left.\pi^{-}\right)$. Therefore, the mass distribution, calculated from the rigidity measurement in the inner tracker and the velocity measured by the TOF, is used to construct the templates and to differentiate between the antiproton signal and the background. The background $e^{-}$and $\pi^{-}$templates are defined from the data sample selected using information from the TRD, the RICH, and also the ECAL, when the event can be measured by the ECAL.

At intermediate rigidities, $\Lambda_{\mathrm{TRD}}$ and the velocity measured with the RICH $\beta_{\text {RICH }}$ are used to separate the antiproton signal from light particles $\left(e^{-}\right.$and $\left.\pi^{-}\right)$. As an example, Fig. 1(a) shows that the antiproton signal and the background are well separated in the $\left(\beta_{\mathrm{RICH}}-\Lambda_{\mathrm{TRD}}\right)$ plane for the absolute rigidity range 5.4-6.5 GV. To determine the number of antiproton signal events, the $\pi^{-}$background is removed by a rigidity dependent $\beta_{\text {RICH }}$ cut and the $\Lambda_{\text {TRD }}$ distribution is used to construct the templates and to differentiate between the $\bar{p}$ signal and $e^{-}$background. The background template is defined from the $e^{-}$data sample selected using ECAL. The Monte Carlo simulation matches the data for $e^{-}$events inside the ECAL. The Monte Carlo simulation was then used to verify that the $e^{-}$ template shape outside the ECAL and inside the ECAL are identical.
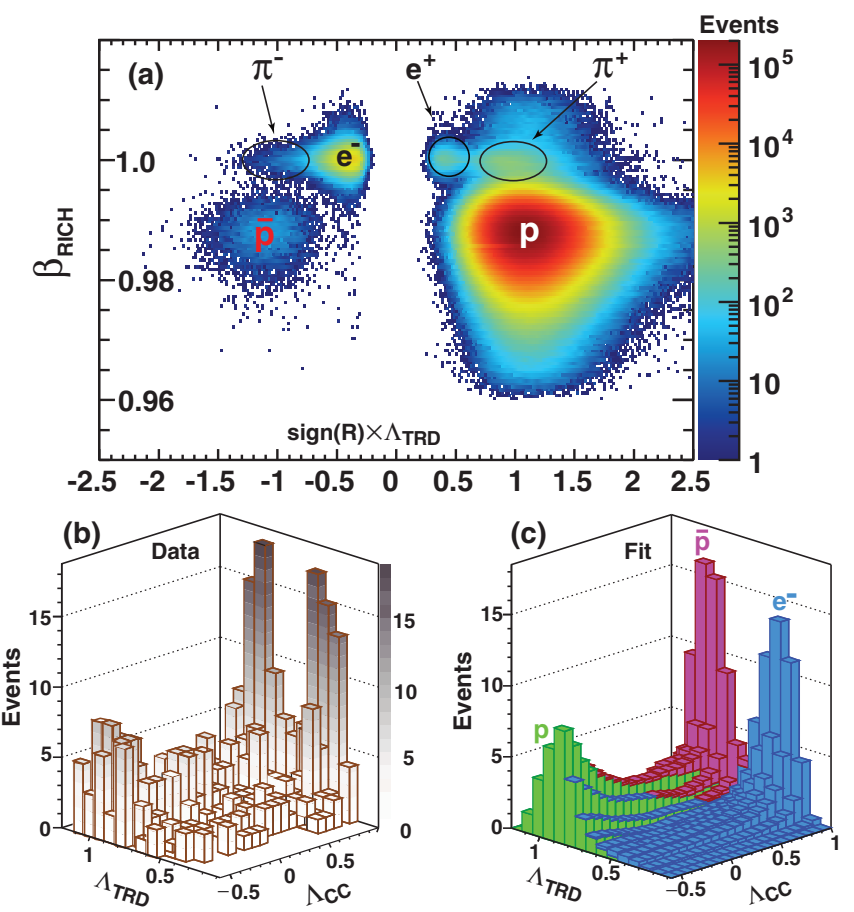

FIG. 1. (a) Negative rigidity and positive rigidity data samples in the $\left[\beta_{\mathrm{RICH}}-\operatorname{sign}(R) \times \Lambda_{\mathrm{TRD}}\right]$ plane for the absolute rigidity range 5.4-6.5 GV. The contributions of $\bar{p}, p, e^{+}, e^{-}, \pi^{+}$, and $\pi^{-}$ are clearly seen. The antiproton signal is well separated from the backgrounds. (b) For negative rigidity events, the distribution of data events in the $\left(\Lambda_{\mathrm{TRD}}-\Lambda_{\mathrm{CC}}\right)$ plane for the absolute rigidity bin $175-211$ GV. (c) Fit with $\chi^{2} /$ d.o.f. $=138 / 154$ of the antiproton signal template (magenta), the electron background template (blue), and the charge confusion proton background template (green) to the data in (b).

In the high rigidity region, the two-dimensional $\left(\Lambda_{\mathrm{TRD}}-\Lambda_{\mathrm{CC}}\right)$ distribution is used to determine the number of antiproton signal events. The lower bound of $\Lambda_{\mathrm{CC}}$ is chosen for each bin to optimize the accuracy of the fit. For example, for $|R|>175 \mathrm{GV}, \Lambda_{\mathrm{CC}} \geq-0.6$. Variation of the lower bound is included in the systematic errors discussed below. To fit the data three template shapes are defined. The first two are for antiprotons and electrons with correctly reconstructed charge sign and the last one is for charge confusion protons. The background templates (i.e., electrons and charge confusion protons) are from the Monte Carlo simulation. The Monte Carlo simulation of the change confusion was verified with the $400 \mathrm{GV}$ proton test beam data. An example of the fit to the data is shown in Figs. 1(b) and 1(c) for the rigidity bin 175-211 GV. The distribution of data in the $\left(\Lambda_{\mathrm{TRD}}-\Lambda_{\mathrm{CC}}\right)$ plane is shown in Fig. 1(b) and the fit results showing the signal and background distributions is highlighted in Fig. 1(c). The $\chi^{2}$ of the fit is 138 for 154 degrees of freedom.

Overall, results for all 57 rigidity bins give a total of $3.49 \times 10^{5}$ antiproton events in the data.

Analysis.-The isotropic antiproton flux for the absolute rigidity bin $R_{i}$ of width $\Delta R_{i}$ is given by 


$$
\Phi_{i}^{\bar{p}}=\frac{N_{i}^{\bar{p}}}{A_{i}^{\bar{p}} T_{i} \Delta R_{i}},
$$

where the rigidity is defined on top of the AMS, $N_{i}^{\bar{p}}$ is the number of antiprotons in the rigidity bin $i$ corrected with the rigidity resolution function (see below), $A_{i}^{\bar{p}}$ is the corresponding effective acceptance that includes geometric acceptance as well as the trigger and selection efficiency, and $T_{i}$ is the exposure time.

Detector resolution effects cause migration of events $N_{i}^{\bar{p}}$ from rigidity bin $R_{i}$ to the measured rigidity bins $\tilde{R}_{j}$ resulting in the observed number of events $\tilde{N}_{j}^{\bar{p}}$. To account for this event migration, an iterative unfolding procedure [31] is used to correct the number of observed events. It is described in detail in our publication on the proton flux [16]. At each iteration, the folded acceptance is defined using the Monte Carlo simulation: $\tilde{A}_{i}^{\bar{p}}=\left(1 / \Phi_{i}^{\bar{p}}\right) \sum_{j} \Phi_{j}^{\bar{p}} A_{j}^{\bar{p}} M_{i j}^{\bar{p}}$, where $M_{i j}^{\bar{p}}$ is the migration probability from bin $j$ to bin $i$. Parametrizing $\tilde{A}^{\bar{p}}$ with a spline function, the number of events is corrected bin by bin by a factor $A^{\bar{p}} / \tilde{A}^{\bar{p}}$ and the flux is recalculated according to Eq. (1). The iteration proceeds until the fluxes calculated for two consecutive steps agree within $0.1 \%$ and the measured flux can be expressed as

$$
\Phi_{i}^{\bar{p}}=\frac{\tilde{N}_{i}^{\bar{p}}}{\tilde{A}_{i}^{\bar{p}} T_{i} \Delta R_{i}} .
$$

The same procedure is used to unfold the observed number of $2.42 \times 10^{9}$ proton events in this analysis.

The $(\bar{p} / p)$ flux ratio is defined for each absolute rigidity bin by

$$
\left(\frac{\bar{p}}{p}\right)_{i} \equiv \frac{\Phi_{i}^{\bar{p}}}{\Phi_{i}^{p}}=\frac{\tilde{N}_{i}^{\bar{p}}}{\tilde{N}_{i}^{p}} \frac{\tilde{A}_{i}^{p}}{\tilde{A}_{i}^{\bar{p}}} .
$$

With $3.49 \times 10^{5}$ antiproton events, the detailed study of systematic errors of the $\bar{p}$ flux and $(\bar{p} / p)$ flux ratio is the key part of the present analysis.

There are four sources of systematic errors on the $\bar{p}$ flux and $(\bar{p} / p)$ flux ratio. The first source affects mostly $\tilde{N}_{i}^{\bar{p}}$ and, to a much lesser extent, $\tilde{N}_{i}^{p}$. It includes uncertainties in the definition of the geomagnetic cutoff factor, in the event selection, and in the shape of the templates. The second source affects $\tilde{A}_{i}^{\bar{p}}$ and $\tilde{A}_{i}^{p}$. It includes uncertainties in the inelastic cross sections of protons and antiprotons in the detector materials and in the migration matrices $M_{i j}^{\bar{p}}$ and $M_{i j}^{p}$. The third source is the uncertainty in the absolute rigidity scale. The fourth source, relevant only for the $\bar{p}$ flux, is the uncertainty in the normalization of the effective folded acceptance $\tilde{A}_{i}^{\bar{p}}$. Contributions of these four sources to the systematic errors on the $\bar{p}$ flux and $(\bar{p} / p)$ flux ratio are discussed below. They are added quadratically to arrive at the systematic errors.

Variation of the geomagnetic cutoff factor in the range 1.2 to 1.4 shows a systematic uncertainty of $\sim 1 \%$ at $1 \mathrm{GV}$ and negligible above $2 \mathrm{GV}$ for both $\tilde{N}_{i}^{\bar{p}}$ and $\tilde{N}_{i}^{p}$. To evaluate systematic uncertainties related to the event selection, the analysis is repeated in each rigidity bin $\sim 1000$ times with different sets of cut values, such that the selection efficiency varies up to $10 \%$ (see Ref. [11] for details). This uncertainty in $\tilde{N}_{i}^{\bar{p}}$ amounts to $4 \%$ at $1 \mathrm{GV}, 0.5 \%$ at $10 \mathrm{GV}$, and rises to $6 \%$ at $450 \mathrm{GV}$. The uncertainty in $\tilde{N}_{i}^{p}$ is negligible over the entire rigidity range.

Uncertainty in the shape of the fit templates affects only $\tilde{N}_{i}^{\bar{p}}$. It becomes particularly important at high rigidities $(>150 \mathrm{GV})$ where charge confusion protons enter the antiproton region. Three template shapes are used for the fit in this region-the antiproton signal template, the electron background template, and the charge confusion proton template. The antiproton signal template has the same shape as protons reconstructed with correct charge-sign (see above). It is extracted from high statistics proton data, therefore the systematic effects are negligible. The electron template from the Monte Carlo simulation is validated with electron data and does not contribute to the systematic error. The systematic error due to the uncertainty in the shape of the charge confusion proton template originates from the uncertainties of the proton flux in the TV region and from the uncertainties of the proton rigidity resolution function. The former is estimated by varying the spectral index of the proton flux within the accuracy of our proton measurement [16]. The later is estimated by comparing the charge confusion amount predicted by the Monte Carlo simulation with the one obtained from the fit of the three template shapes to the negative rigidity data. Overall, the systematic error from the templates is estimated to be $12 \%$ at $450 \mathrm{GV}$, decreasing to $<1 \%$ below $30 \mathrm{GV}$.

The systematic errors on the folded acceptances $\tilde{A}^{\bar{p}}$ and $\tilde{A}^{p}$ originate from the uncertainties in the interaction cross sections for protons and antiprotons in the detector materials $[32,33]$ and the uncertainties in the migration matrices $M_{i j}^{\bar{p}}$ and $M_{i j}^{p}$. The systematic error from the cross section uncertainties is estimated by varying the $\bar{p}$ and $p$ interaction cross sections in the Monte Carlo simulation within the accuracy of the cross section measurements $[32,33]$. The corresponding systematic error on $\tilde{A}^{\bar{p}}$ is found to be $4 \%$ at $1 \mathrm{GV}$ and $\sim 1 \%$ above $50 \mathrm{GV}$. The error on $\tilde{A}^{p}$ is found to be $2.5 \%$ at $1 \mathrm{GV}$ and $\sim 1 \%$ above $50 \mathrm{GV}$. These values are larger than those in Ref. [16] due to the larger acceptance. The systematic errors on $\tilde{A}^{\bar{p}}$ and $\tilde{A}^{p}$ due to the cross section uncertainties are independent and they are added in quadrature to get the systematic error on the $\tilde{A}^{p} / \tilde{A}^{\bar{p}}$ ratio. The systematic uncertainty in the migration matrix $M_{i j}^{p}$ is studied by comparing the test beam data at 
$400 \mathrm{GV}$ with the Monte Carlo simulation. The rigidity resolution function $\Delta(1 / R)$ has a pronounced Gaussian core of width $\sigma$ and non-Gaussian tails spanning beyond $2.5 \sigma$ from the center [16]. Uncertainties in the core and in the tails of the antiproton migration matrix $M_{i j}^{\bar{p}}$ are assumed to be the same as for $M_{i j}^{p}$. Varying both the width of the core and the amount of non-Gaussian tails as described in Ref. [16] yields the systematic error of $1 \%$ below $200 \mathrm{GV}$ and $1.5 \%$ at $450 \mathrm{GV}$ for both $\tilde{A}^{\bar{p}}$ and $\tilde{A}^{p}$. These systematic errors partially cancel in the $\tilde{A}^{p} / \tilde{A}^{\bar{p}}$ ratio, yielding uncertainties of $1 \%$ at $1 \mathrm{GV}$ and $<0.5 \%$ above $2 \mathrm{GV}$. We note that the $\tilde{A}^{p} / \tilde{A}^{\bar{p}}$ ratio decreases from 1.15 at $1 \mathrm{GV}$ to 1.04 at $450 \mathrm{GV}$ due to the varying difference of interaction cross sections for protons and antiprotons, and due to bin-to-bin event migration.

The error on the absolute rigidity scale due to the residual misalignment of the tracker planes was estimated by comparing the electron and positron energies measured in the ECAL with the momentum measured in the tracker to be $1 / 26 \mathrm{TV}^{-1}$, see Ref. [16] for details. The corresponding errors on the antiproton and proton fluxes are negligble below $10 \mathrm{GV}$ and gradually increase to $\sim 1 \%$ at $450 \mathrm{GV}$. This error has opposite effects on the measured proton and antiproton fluxes, therefore the error on the $(\bar{p} / p)$ flux ratio gradually increases to $\sim 2 \%$ at $450 \mathrm{GV}$.

The systematic error on the normalization of the effective folded acceptance is due to small differences between the data and the Monte Carlo samples [16]. This error is relevant only for the $\bar{p}$ flux; it cancels in the $(\bar{p} / p)$ flux ratio. It is estimated to be $5 \%$ at $1 \mathrm{GV}$ decreasing gradually to $2 \%$ above $20 \mathrm{GV}$.

As stated above, charge confusion protons are protons which are reconstructed in the tracker with negative rigidity due to the finite tracker resolution or due to interactions with the detector materials. The uncertainty from the charge confusion proton template becomes significant for $|R|>30 \mathrm{GV}$. To ensure that the shape of the charge confusion proton background templates from the Monte Carlo simulation does not introduce bias into the antiproton identification, we also performed a completely independent data driven analysis based on the linear regression method [34] for $|R|>30 \mathrm{GV}$ over the acceptance which includes L1, L9, and ECAL. In this analysis a weighted sum is constructed from a set of measured quantities sensitive to the finite tracker resolution and interactions with the detector materials. This set includes information from the TRD, TOF, tracker, ACC, and ECAL. In particular, the tracker information includes the $\chi^{2} /$ d.o.f., the rigidity reconstructed using different combinations of tracker layers, and the maximum distance of the tracker hits from the fitted track. The weights of individual quantities are optimized to maximize the separation between the antiproton signal and the charge confusion background. The resultant sums are nearly Gaussian for both the antiproton signal and the charge confusion proton background. This allows construction of the template (i.e., a Gaussian distribution with $\sim 1 \%$ non-Gaussian tails) without relying on the Monte Carlo simulation. The non-Gaussian tails are evaluated using iterative fits with the subtraction of both the antiproton and charge confusion proton templates from the negative rigidity data. The details of the analysis used in this technique will be presented in a separate publication. The results of this data driven analysis agree within the systematic errors with those presented in this Letter.

Most importantly, in addition to the linear regression analysis, several other independent analyses were performed on the same data sample by different study groups. The results of those analyses are consistent with this Letter.

Results.-The measured antiproton flux and antiproton-toproton flux ratio together with their statistical and systematic errors are presented in Table I of Supplemental Material [18] as a function of the absolute rigidity value at the top of the AMS detector along with the number of observed antiproton events $\tilde{N}^{\bar{p}}$. The statistical errors are from the fit errors on the signal. As seen from this table, the statistical and systematic error contributions to the total error in the flux and flux ratio vary with rigidity. For $1.00 \leq|R|<1.33 \mathrm{GV}$ the statistical error dominates, for $1.33 \leq|R|<1.71 \mathrm{GV}$ the errors are comparable, for $1.71 \leq|R|<48.5 \mathrm{GV}$ the systematic error dominates, for $48.5 \leq|R|<108 \mathrm{GV}$ the errors are comparable, and for $108 \leq|R|<450 \mathrm{GV}$ statistical error dominates.

AMS has now measured the fluxes of electrons and positrons [15] and of protons [16] with the same detector and same time period, May 19, 2011 to November 26, 2013. The antiprotons in this Letter were measured from May 19, 2011 to May 26, 2015. We have studied the time dependent solar effects on these fluxes during the interval covered by this Letter [35]. Within our current accuracy, the time dependent solar effects are observable for antiprotons, electrons, and positrons with $|R|<10 \mathrm{GV}$ and for protons with $|R|<20 \mathrm{GV}$. For the study of the flux ratio dependence of elementary particles (see below), we chose $|R|>$ $10 \mathrm{GV}$ where the time dependent solar effects for protons are small and the uncertainties are dominated by the accuracies of the measurements of electrons, positrons, and antiprotons. This enables us to study the overall rigidity dependent behavior of different fluxes as shown in Fig. 2 above $10 \mathrm{GV}$. The points are placed along the abscissa at $\hat{R}$ calculated for a flux $\propto|R|^{-2.7}$ [36]. As seen from Fig. 2, the rigidity dependence of the fluxes for antiprotons, positrons, and protons are nearly identical above $\sim 60 \mathrm{GV}$ whereas the rigidity dependence of the electron flux is different.

Analysis of the antiproton spectral index $\gamma_{\bar{p}}$ is performed over independent rigidity intervals with a variable width to have sufficient sensitivity to $\gamma_{\bar{p}}$. The spectral index is calculated from $\gamma_{\bar{p}}=d\left[\log \left(\Phi^{\bar{p}}\right)\right] / d[\log (|R|)]$. The result is presented in Fig. 3 of Supplemental Material [18] compared with our result on the proton spectral index [16]. As seen, over the four lowest rigidity points, $|R|<60.3 \mathrm{GV}$, 


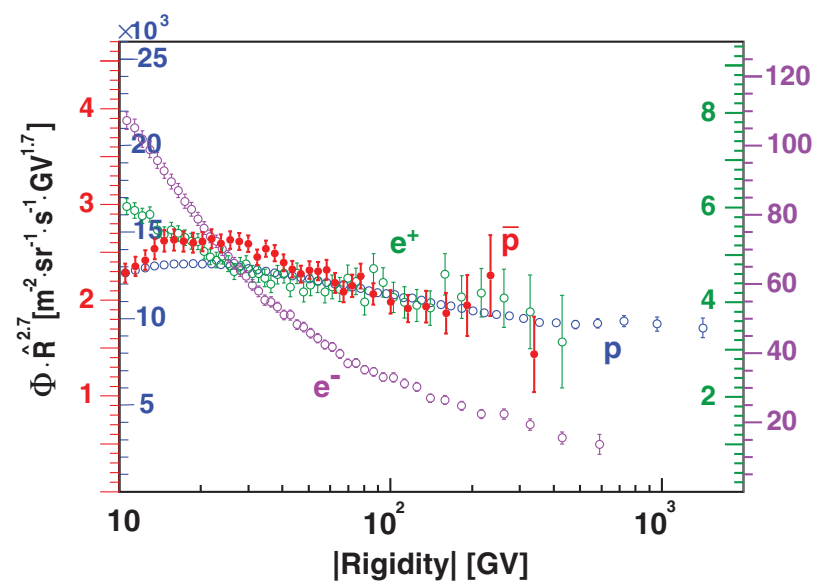

FIG. 2. The measured antiproton flux (red, left axis) compared to the proton flux (blue, left axis) [16], the electron flux (purple, right axis), and the positron flux (green, right axis) [15]. All the fluxes are multiplied by $\hat{R}^{2.7}$. The fluxes show different behavior at low rigidities while at $|R|$ above $\sim 60 \mathrm{GV}$ the functional behavior of the antiproton, proton, and positron fluxes are nearly identical and distinctly different from the electron flux. The error bars correspond to the quadratic sum of the statistical and systematic errors.

the antiproton spectral index decreases more rapidly than the proton spectral index and for the highest rigidity interval, $60.3 \leq|R|<450 \mathrm{GV}$, the antiproton spectral index is consistent with the proton spectral index.

Figure 3(a) presents the measured $(\bar{p} / p)$ flux ratio. Compared with earlier experiments [2,6], the AMS results extend the rigidity range to $450 \mathrm{GV}$ with increased precision. Figure 2 of Supplemental Material [18] shows the low energy $(<10 \mathrm{GeV})$ part of our measured $(\bar{p} / p)$ flux ratio. To minimize the systematic error for this flux ratio we have used the $2.42 \times 10^{9}$ protons selected with the same acceptance, time period, and absolute rigidity range as the antiprotons. From 10 to $450 \mathrm{GV}$, the values of the proton flux are identical to $1 \%$ to those in our publication [16]. As seen from Fig. 3(a), above $\sim 60 \mathrm{GV}$ the ratio appears to be rigidity independent.

To estimate the lowest rigidity above which the $(\bar{p} / p)$ flux ratio is rigidity independent, we use rigidity intervals with starting rigidities from $10 \mathrm{GV}$ and increasing bin by bin. The ending rigidity for all intervals is fixed at $450 \mathrm{GV}$. Each interval is split into two sections with a boundary between the starting rigidity and $450 \mathrm{GV}$. Each of the two sections is fit with a constant and we obtain two mean values of the $(\bar{p} / p)$ flux ratio. The lowest starting rigidity of the interval that gives consistent mean values at the 90\% C.L. for any boundary defines the lowest limit. This yields $60.3 \mathrm{GV}$ as the lowest rigidity above which the $(\bar{p} / p)$ flux ratio is rigidity independent with a mean value of $(1.81 \pm 0.04) \times 10^{-4}$. To further probe the behavior of the flux ratio we define the best straight line fit over a rigidity interval as

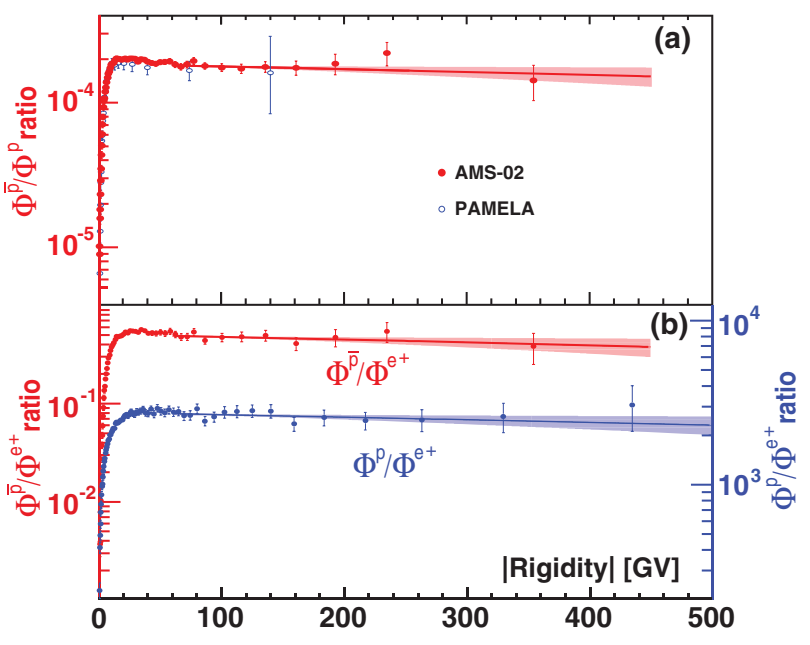

FIG. 3. (a) The measured $(\bar{p} / p)$ flux ratio as a function of the absolute value of the rigidity from 1 to $450 \mathrm{GV}$. The PAMELA [6] measurement is also shown. (b) The measured $\left(\bar{p} / e^{+}\right)$(red, left axis) and $\left(p / e^{+}\right)$(blue, right axis) flux ratios. The solid lines show the best fit of Eq. (4) to the data above the lowest rigidity consistent with rigidity independence together with the $68 \%$ C.L. ranges of the fit parameters (shaded regions). For the AMS data, the error bars are the quadratic sum of statistical and systematic errors. Horizontally, the data points are placed at the center of each bin.

$$
(\bar{p} / p)=C+k\left(|R|-R_{0}\right),
$$

where $C$ is the value of the flux ratio at $R_{0}, k$ is the slope, and $R_{0}$ is chosen to minimize the correlation between the fitted values of $C$ and $k$, i.e., the mean of $|R|$ over the interval weighted with the statistical and uncorrelated systematic errors. The solid red line in Fig. 3(a) shows this best straight line fit above $60.3 \mathrm{GV}$, as determined above, together with the $68 \%$ C.L. range of the fit parameters (shaded region). Above $60.3 \mathrm{GV}, R_{0}=91 \mathrm{GV}$. The fitted value of the slope, $k=(-0.7 \pm 0.9) \times 10^{-7} \mathrm{GV}^{-1}$, is consistent with zero.

With the AMS measurements on the fluxes of all charged elementary particles in cosmic rays, $\bar{p}, p, e^{+}$, and $e^{-}$, we can now study the rigidity dependent behavior of different flux ratios. The flux ratios and errors are tabulated in Tables II and III of Supplemental Material [18]. For the antiprotonto-positron ratio the rigidity independent interval is $60.3 \leq$ $|R|<450 \mathrm{GV}$ with a mean value of $0.479 \pm 0.014$. Fitting Eq. (4) over this interval yields $k\left(\bar{p} / e^{+}\right)=(-2.8 \pm 3.2) \times$ $10^{-4} \mathrm{GV}^{-1}$. For the proton-to-positron ratio, the rigidity independent interval is $59.13 \leq|R|<500 \mathrm{GV}$ with a mean value of $(2.67 \pm 0.05) \times 10^{3}$ and $k\left(p / e^{+}\right)=(-0.9 \pm$ 1.0) $\mathrm{GV}^{-1}$. Both results are shown in Fig. 3(b) together with the $68 \%$ C.L. range of the fit parameters (shaded regions). In the study of the ratios, we have taken into account the correlation of the errors due to uncertainty in the ECAL energy scale in $\Phi^{e^{ \pm}}$[15].

In Fig. 4 of Supplemental Material [18] we present our measured antiproton-to-electron and proton-to-electron flux ratios. Both of these flux ratios exhibit rigidity 


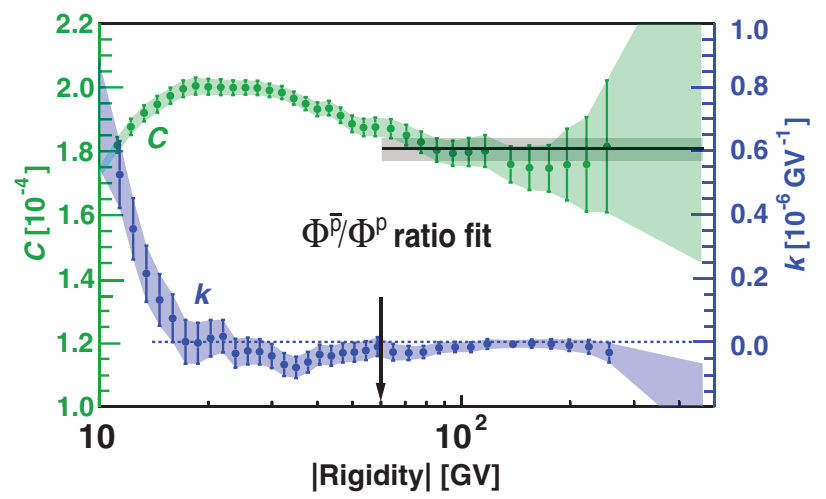

FIG. 4. Sliding window fits of Eq. (4) to the $(\bar{p} / p)$ flux ratio measured by AMS with parameter $C$ (green, left axis) and the slope $k$ (blue, right axis). The green and blue shaded regions indicate that the errors are correlated between adjacent points. The points are placed at $R_{0}$. The dashed blue line at $k=0$ is to guide the eye. The black arrow indicates the lowest rigidity above which the flux ratio is consistent with being rigidity independent and the black horizontal band shows the mean value and the 1-sigma error of the flux ratio above this rigidity.

behavior which is distinct from that observed in the antiproton-to-proton, antiproton-to-positron, and protonto-positron flux ratios.

To examine the rigidity dependence of the flux ratios shown in Figs. 3(a) and 3(b) quantitatively in a model independent way, Eq. (4) is fit to the flux ratios over their rigidity ranges with a sliding window. For each flux ratio, the width of the window varies with rigidity to have sufficient sensitivity to the slope $k$ such that each window covers between four and eight bins. The variations of $C$ and slope $k$ for the $(\bar{p} / p)$ flux ratio are shown in Fig. 4. At low rigidity the slope $k$ crosses zero, that is, the ratio reaches a maximum at $\sim 20 \mathrm{GV}$ as also clearly seen in the parameter $C$. As seen from Fig. 5 of Supplemental Material [18], the rigidity dependence of the $\left(\bar{p} / e^{+}\right)$and $\left(p / e^{+}\right)$flux ratios are nearly identical to that of the $(\bar{p} / p)$ flux ratio. Also shown in Fig. 4, as well as in Fig. 5 of the Supplemental Material [18], are the mean values of the flux ratios over the intervals where they are rigidity independent.

In conclusion, with this measurement of the antiproton flux and the $(\bar{p} / p)$ flux ratio, AMS has simultaneously measured all the charged elementary particle cosmic ray fluxes and flux ratios. In the absolute rigidity range $\sim 60$ to $\sim 500 \mathrm{GV}$, the antiproton, proton, and positron fluxes are found to have nearly identical rigidity dependence and the electron flux exhibits a different rigidity dependence. In the absolute rigidity range below $60 \mathrm{GV}$, the $(\bar{p} / p),\left(\bar{p} / e^{+}\right)$, and $\left(p / e^{+}\right)$flux ratios each reaches a maximum. In the absolute rigidity range $\sim 60$ to $\sim 500 \mathrm{GV}$, the $(\bar{p} / p)$, $\left(\bar{p} / e^{+}\right)$, and $\left(p / e^{+}\right)$flux ratios show no rigidity dependence. These are new observations of the properties of elementary particles in the cosmos.

We thank former NASA Administrator Daniel S. Goldin for his dedication to the legacy of the ISS as a scientific laboratory and his decision for NASA to fly AMS as a DOE payload. We also acknowledge the continuous support of the NASA leadership including Charles Bolden and William H. Gerstenmaier and of the JSC and MSFC flight control teams which has allowed AMS to operate optimally on the ISS for five years. We are grateful for the support of Jim Siegrist and his staff of the DOE. We also acknowledge the continuous support from MIT and its School of Science, Michael Sipser, Marc Kastner, Ernest Moniz, Richard Milner, and Boleslaw Wyslouch. Research supported by São Paulo Research Foundation (FAPESP) Grants No. 2014/19149-7, No. 2014/50747-8, and No. 2015-50378-5, Brazil; CAS, NSFC, MOST, NLAA, the provincial governments of Shandong, Jiangsu, Guangdong, and the China Scholarship Council, China; the Finnish Funding Agency for Innovation (Tekes) Grants No. 40361/01 and No. 40518/03 and the Academy of Finland Grant No. 258963, Finland; CNRS, IN2P3, CNES, Enigmass, and the ANR, France; Pascale Ehrenfreund, DLR, and JARA-HPC under Project No. JARA0052, Germany; INFN and ASI under ASI-INFN Agreements No. 2013002-R.0 and No. 2014-037-R.0, Italy; CHEP Grants No. NRF-2009-0080142 and No. NRF-2012-010226 at Kyungpook National University and No. NRF-2013004883 at Ewha Womans University, Korea; the Consejo Nacional de Ciencia y Tecnología and UNAM, Mexico; FCT under Grant No. PTDC/FIS/122567/2010, Portugal; CIEMAT, IAC, CDTI, and SEIDI-MINECO under Grants No. AYA2012-39526-C02-(01/02), No. ESP2015-71662-C2-(1-P/2-P), No. SEV-2011-0187, No. SEV-2015-0548, and No. MDM-2015-0509, Spain; the Swiss National Science Foundation (SNSF), federal and cantonal authorities, Switzerland; Academia Sinica and the Ministry of Science and Technology (MOST) under Grants No. 103-2112-M-006-018-MY3, No. 1042112-M-001-027, and No. CDA-105-M06, former President of Academia Sinica Yuan-Tseh Lee, and former Ministers of MOST Maw-Kuen Wu and Luo-Chuan Lee, Taiwan; the Turkish Atomic Energy Authority at METU, Turkey; and NSF Grant No. 1455202, Wyle Laboratories Grant No. 2014/T72497, and NASA NESSF Grant No. HELIO15F-0005, USA. We gratefully acknowledge the strong support from CERN including Rolf-Dieter Heuer and Fabiola Gianotti, from the CERN IT department and Bernd Panzer-Steindel, and from the European Space Agency including Johann-Dietrich Wörner and Simonetta Di Pippo. We are grateful for important discussions with Fiorenza Donato, Jonathan Ellis, Jonathan Feng, Igor Moskalenko, Michael Salamon, Subir Sarkar, Joachim Trümper, Michael S. Turner, Steven Weinberg, and Arnold Wolfendale.

\footnotetext{
${ }^{a}$ Also at ASI, I-00133 Roma, Italy.

${ }^{b}$ Present address: Departamento de Ciencias, Pontifica Universidad Católica del Perú (PUCP), Lima 32, Peru.
} 
${ }^{c}$ Also at ASI Science Data Center (ASDC), I-00133 Roma, Italy.

${ }^{\mathrm{d}}$ Also at Sun Yat-Sen University (SYSU), Guangzhou, 510275, China.

${ }^{\mathrm{e}}$ Also at Wuhan University, Wuhan 430072, China.

${ }^{\mathrm{f}}$ Also at Harbin Institute of Technology (HIT), Harbin 150001, China.

${ }^{\mathrm{g}}$ Also at Huazhong University of Science and Technology (HUST), Wuhan, 430074, China.

${ }^{\mathrm{h}}$ Also at Università di Siena, I-53100 Siena, Italy.

iAlso at Laboratoire d'Annecy-le-Vieux de Physique Théorique (LAPTh), CNRS and Université Savoie Mont Blanc, F-74941 Annecy-le-Vieux, France.

${ }^{\mathrm{j}}$ Also at Shandong University (SDU), Jinan, Shandong, 250100, China.

${ }^{\mathrm{k}}$ Also at Nankai University, Tianjin 300071, China.

${ }^{1}$ Also at Southeast University (SEU), Nanjing, 210096, China.

${ }^{\mathrm{m}}$ Also at Institute of Theoretial Physics, Chinese Academy of Sciences, Beijing 100190, China.

${ }^{\mathrm{n}}$ Also at Jilin University, Jilin 130012, China.

[1] E. A. Bogomolov et al., in Proceedings of the 16th International Cosmic Ray Conference, University of Tokio, Tokio, Japan (1979) Vol. 1, p. 330; R. L. Golden, S. Horan, B. G. Mauger, G. D. Badhwar, J. L. Lacy, S. A. Stephens, R. R Daniel, and J. E. Zipse, Phys. Rev. Lett. 43, 1196 (1979); A. Buffington and S. M. Shindler, Astrophys. J. 247, L105 (1981).

[2] K. Yoshimura et al., Phys. Rev. Lett. 75, 3792 (1995); S. Orito et al., Phys. Rev. Lett. 84, 1078 (2000); Y. Asaoka et al., Phys. Rev. Lett. 88, 051101 (2002); K. Abe et al., Phys. Lett. B 670, 103 (2008); Phys. Rev. Lett. 108, 051102 (2012); Astrophys. J. 822, 65 (2016).

[3] M. Boezio et al., Astrophys. J. 487, 415 (1997); 561, 787 (2001).

[4] A. S. Beach et al., Phys. Rev. Lett. 87, 271101 (2001).

[5] M. Aguilar et al., Phys. Rep. 366, 331 (2002).

[6] O. Adriani et al., Phys. Rev. Lett. 102, 051101 (2009); 105, 121101 (2010); JETP Lett. 96, 621 (2013).

[7] C. S. Shen and G. B. Berkey, Phys. Rev. 171, 1344 (1968); I. V. Moskalenko and A. W. Strong, Astrophys. J. 493, 694 (1998); M. Simon, A. Molnar, and S. Roesler, Astrophys. J. 499, 250 (1998); I. V. Moskalenko, A. W. Strong, J. F. Ormes, and M.S. Potgieter, Astrophys. J. 565, 280 (2002); V. S. Ptuskin, I. V. Moskalenko, F. C. Jones, A. W. Strong, and V. N. Zirakashvili, Astrophys. J. 642, 902 (2006); F. Donato, D. Maurin, P. Brun, T. Delahaye, and P. Salati, Phys. Rev. Lett. 102, 071301 (2009).

[8] G. Jungman, M. Kamionkowski, and K. Griest, Phys. Rep. 267, 195 (1996); L. Bergström, Rep. Prog. Phys. 63, 793 (2000); G. Bertone, D. Hooper, and J. Silk, Phys. Rep. 405, 279 (2005); G. Kane, R. Lu, and S. Watson, Phys. Lett. B 681, 151 (2009); F. Donato, D. Maurin, P. Brun, T. Delahaye, and P. Salati, Phys. Rev. Lett. 102, 071301 (2009).

[9] P. Blasi and P. D. Serpico, Phys. Rev. Lett. 103, 081103 (2009); Y. Fujita, K. Kohri, R. Yamazaki, and K. Ioka, Phys. Rev. D 80, 063003 (2009).

[10] S. W. Hawking, Nature (London) 248, 30 (1974); P. Kiraly, J. Szabelski, J. Wdowczyk, and A. W. Wolfendale, Nature (London) 293, 120 (1981).
[11] M. Aguilar et al., Phys. Rev. Lett. 110, 141102 (2013); L. Accardo et al., Phys. Rev. Lett. 113, 121101 (2014).

[12] J. Kopp, Phys. Rev. D 88, 076013 (2013); L. Feng, R-Z. Yang, H-N. He, T-K. Dong, Y-Z. Fan, and J. Chang, Phys. Lett. B 728, 250 (2014); L. Bergström, T. Bringmann, I. Cholis, D. Hooper, and C. Weniger, Phys. Rev. Lett. 111, 171101 (2013); C. H. Chen, C. W. Chiang, and T. Nomura, Phys. Lett. B 747, 495 (2015); H. B. Jin, Y. L. Wu, and Y.-F. Zhou, Phys. Rev. D 92, 055027 (2015).

[13] I. Cholis and D. Hooper, Phys. Rev. D 88, 023013 (2013); T. Linden and S. Profumo, Astrophys. J. 772, 18 (2013); P. Mertsch and S. Sarkar, Phys. Rev. D 90, 061301 (2014); K. Kohri, K. Ioka, Y. Fujita, and R. Yamazaki, Prog. Theor. Exp. Phys. 2016, 021E01 (2016).

[14] K. Blum, B. Katz, and E. Waxman, Phys. Rev. Lett. 111, 211101 (2013); R. Cowsik, B. Burch, and T. MadziwaNussinov, Astrophys. J. 786, 124 (2014); R. Kappl and M. W. Winkler, J. Cosmol. Astropart. Phys. 09 (2014) 051.

[15] M. Aguilar et al., Phys. Rev. Lett. 113, 121102 (2014).

[16] M. Aguilar et al., Phys. Rev. Lett. 114, 171103 (2015).

[17] A. Kounine, Int. J. Mod. Phys. E 21, 1230005 (2012); S. Rosier-Lees, in Proceedings of the 19th Symposium on Astroparticle Physics in the Netherlands, Beekbergen, 2014 (unpublished); S. C. C. Ting, Nucl. Phys. B, Proc. Suppl. 243-244, 12 (2013); S. C. Lee, in Proceedings of the 20th International Conference on Supersymmetry and Unification of Fundamental Interactions (SUSY 2012), Beijing, 2012 (unpublished); M. Aguilar, in Proceedings of the XL International Meeting on Fundamental Physics, Centro de Ciencias de Benasque Pedro Pascual, 2012 (unpublished); S. Schael, in Proceedings of the 10th Symposium on Sources and Detection of Dark Matter and Dark Energy in the Universe, Los Angeles, 2012 (unpublished); B. Bertucci, Proc. Sci., EPS-HEP (2011) 67; M. Incagli, AIP Conf. Proc. 1223, 43 (2010); R. Battiston, Nucl. Instrum. Methods Phys. Res., Sect. A 588, 227 (2008).

[18] See Supplemental Material at http://link.aps.org/ supplemental/10.1103/PhysRevLett.117.091103 for details of the AMS detector, its test beam calibration, and the simulation; for the tabulated $\bar{p}$ flux $\Phi^{\bar{p}}$ and the $(\bar{p} / p)$, $\left(\bar{p} / e^{+}\right),\left(\bar{p} / e^{-}\right),\left(p / e^{+}\right)$, and $\left(p / e^{-}\right)$flux ratios; and for figures of an antiproton event display, the low energy antiproton-to-proton flux ratio, the antiproton spectral index, the $\left(\bar{p} / e^{-}\right)$and $\left(p / e^{-}\right)$flux ratios, and sliding window fit parameters of the $\left(\bar{p} / e^{+}\right)$and $\left(p / e^{+}\right)$flux ratios.

[19] B. Alpat et al., Nucl. Instrum. Methods Phys. Res., Sect. A 613, 207 (2010).

[20] K. Lübelsmeyer et al., Nucl. Instrum. Methods Phys. Res., Sect. A 654, 639 (2011).

[21] V. Bindi et al., Nucl. Instrum. Methods Phys. Res., Sect. A 743, 22 (2014), and references therein.

[22] Ph. von Doetinchem, W. Karpinski, Th. Kirn, K. Lübelsmeyer, St. Schael, and M. Wlochal, Nucl. Phys. B, Proc. Suppl. 197, 15 (2009).

[23] F. Hauler et al., IEEE Trans. Nucl. Sci. 51, 1365 (2004); Ph. Doetinchem et al., Nucl. Instrum. Methods Phys. Res., Sect. A 558, 526 (2006); Th. Kirn, Nucl. Instrum. Methods Phys. Res., Sect. A 706, 43 (2013).

[24] M. Aguilar-Benitez et al., Nucl. Instrum. Methods Phys. Res., Sect. A 614, 237 (2010); F. Giovacchini, 
Nucl. Instrum. Methods Phys. Res., Sect. A 766, 57 (2014).

[25] C. Adloff et al., Nucl. Instrum. Methods Phys. Res., Sect. A 714, 147 (2013).

[26] B. P. Roe, H. J. Yang, J. Zhu, Y. Liu, I. Stancu, and G. McGregor, Nucl. Instrum. Methods Phys. Res., Sect. A 543, 577 (2005).

[27] J. Alcaraz et al., Phys. Lett. B 484, 10 (2000).

[28] C. C. Finlay et al., Geophys. J. Int. 183, 1216 (2010); E. Thébault et al., Earth Planets Space 67, 79 (2015).

[29] P. Papini et al., Astrophys. J. 615, 259 (2004).

[30] S. Agostinelli et al., Nucl. Instrum. Methods Phys. Res., Sect. A 506, 250 (2003); J. Allison et al., IEEE Trans. Nucl. Sci. 53, 270 (2006).

[31] A. Kondor, Nucl. Instrum. Methods Phys. Res. 216, 177 (1983); V. Blobel, Report No. DESY-84-118 (1984); G. D’Agostini, Nucl. Instrum. Methods Phys. Res., Sect. A 362, 487 (1995).

[32] T. Bowen, M. Di Corato, W. H. Moore, and G. Tagliaferri, II Nuovo Cimento 9, 908 (1958); G. Bellettini, G. Cocconi, A. N. Diddens, E. Lillethun, G. Matthiae, J. P. Scanlon, and A. M. Wetherell, Nucl. Phys. 79, 609 (1966); S. P. Denisov, S. V. Donskov, Yu. P. Gorin, R. N. Krasnokutsky, A. I. Petrukhin, Yu. D. Prokoshkin, and D. A. Stoyanova,
Nucl. Phys. B61, 62 (1973); A. S. Carroll et al., Phys. Lett. B 80, 319 (1979); J. R. Letaw, R. Silberberg, and C. H. Tsao, Astrophys. J. Suppl. Ser. 51, 271 (1983); N. Abgrall et al., Phys. Rev. C 84, 034604 (2011).

[33] B. Cork, G. R. Lambertson, O. Piccioni, and W. A. Wenzel, Phys. Rev. 107, 248 (1957); J. V. Allaby et al., Yad. Fiz. 12, 538 (1970); R. J. Abrams, R. L. Cool, G. Giacomelli, T. F. Kycia, B. A. Leontic, K. K. Li, A. Lundby, D. N. Michael, and J. Teiger, Phys. Rev. D 4, 3235 (1971); H. Aihara et al., Nucl. Phys. A360, 291 (1981); K. Nakamura, J. Chiba, T. Fujii, H. Iwasaki, T. Kageyama, S. Kuribayashi, T. Sumiyoshi, T. Takeda, H. Ikeda, and Y. Takada, Phys. Rev. Lett. 52, 731 (1984).

[34] X. Yan and X. G. Su, Linear Regression Analysis (World Scientific, Singapore, 2009), ISBN-13 978-981 283410 2; A. Hoecker et al., arXiv:physics/0703039v5.

[35] AMS Collaboration, Measurement of the Time Dependent Solar Modulation of Primary Cosmic Rays Fluxes with the Alpha Magnetic Spectrometer on the International Space Station (to be published).

[36] G. D. Lafferty and T. R. Wyatt, Nucl. Instrum. Methods Phys. Res., Sect. A 355, 541 (1995). We have used Eq. (6) with $\hat{R} \equiv x_{l w}$. 